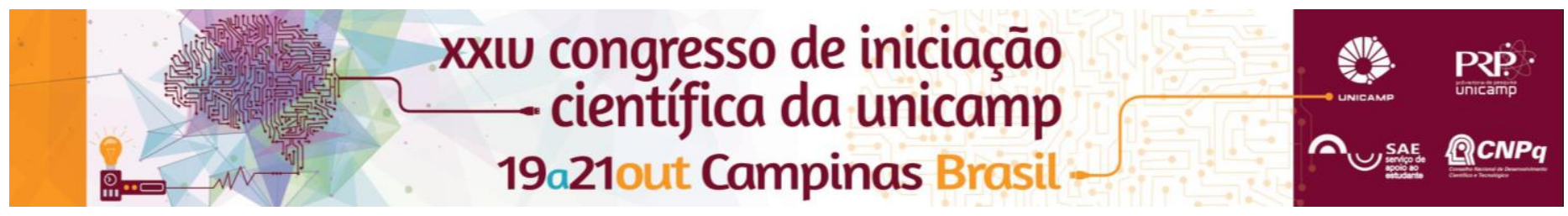

\title{
Wound healing action of topical nitric oxide releasing poly(acrylic acid)/pluronic F127 hydrogel membranes
}

\author{
Lucas Militão*, Carlos P. Jara, Mathilde Champeau, Eliana P. Araújo, Marcelo G. de Oliveira
}

\begin{abstract}
Nitric oxide (NO) plays fundamental roles in wound healing and is endogenously transported by S-nitrosoglutathione (GSNO). In the present work, we incorporated GSNO in hydrogel membranes comprised of poly(acrylic acid)/Pluronic F127 (PAAc/F127/GSNO), with the purpose of delivering $\mathrm{NO}$ topically to accelerate wound healing. Chemiluminescence measurements showed that dry PAAC/F127/GSNO membranes undergo slow hydration with concomitant NO release over more than 9 days. Topical application of the GSNO/PAAc/F127 membranes in an animal wound healing model led to decreased inflammation and increased collagen deposition and organization in the cicatricial tissue, after 21 days. These results point to a potential medical application of these membranes for accelerating wound healing.
\end{abstract}

\section{Key words:}

Nitric Oxide, Hydrogels, Wound healing

\section{Introduction}

Nitric oxide (NO) is an endogenous molecule with several physiological and phatophysiological actions. It has been demonstrated that $\mathrm{NO}$ is involved in both early and proliferative phases of wound healing and regulates collagen formation, cell proliferation and wound contraction $^{[1]}$. S-nitrosoglutathione (GSNO) is an endogenous NO carrier and has been used as an exogenous NO donor in experimental medical applications $^{[2]}$. As a water-soluble molecule, topical, localized applications of GSNO demand the use of hydrophilic matrices, such as hydrogels.

In the present work, we synthesized a hydrogel comprised of a chemically cross linked network of poly(acrylic acid) (PAAc), interpenetrated with a network of micelles of poly(ethylene oxide)-poly(propylene oxide)poly(ethylene oxide) (PEO-PPO-PEO, Pluronic F127). GSNO was incorporated into this membrane by solution absorption technique and the membranes were characterized regarding their morphology, water absorption, GSNO content and spontaneous NO release profile. For a preliminary evaluation of their wound healing action, the PAAc/F127/GSNO membranes were topically applied on lesions in an animal model, using Swiss mice.

\section{Results and Discussion}

Although the The PAAc/F127 membranes displayed a dense morphology with the absence of pores, their water absorption capacity ranged from $84 \mathrm{wt} \%$ in water to $210 w t \%$ in phosphate buffer saline solution at $\mathrm{pH} 7.4$, at $37^{\circ} \mathrm{C}$. This property was used to charge the membranes with aqueous GSNO solution and allowed preparing PAAc/F127 membranes containing up to $4.5 \pm 0,5$ $\mu \mathrm{mol} \mathrm{GSNO}_{\mathrm{GS}} / \mathrm{cm}^{3}$.

The NO release profile from the PAAc/F127/GSNO membranes due to the spontaneous $\mathrm{NO}$ release from GSNO, forming oxidized glutathione, was characterized by chemiluminescence. The membranes were shown to be capable of releasing $\mathrm{NO}$ continuously in a $\mathrm{nmo} / \mathrm{min}$ range, over more than 9 days, under a slow hydration condition. Topical application of the membranes on the wounds of the animals during 21 days led to a reduction in the infllammatory infiltrate and an increase in the collagen deposition and collagen fibers organization in the cicatricial tissue, compared to the control group (Fig. 1).
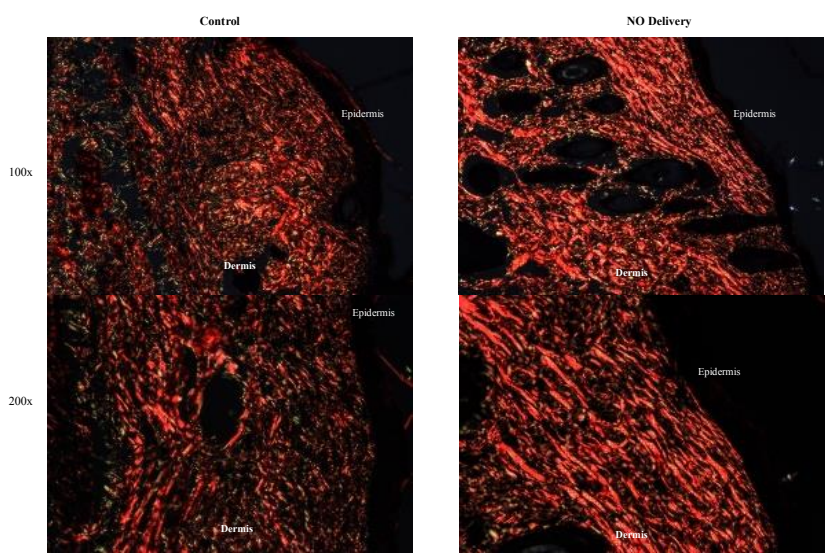

Fig. 1. Representative picro-sirius red-stained sections of the wound tissue of animals under polarized light 21 after wounding. Left: control animals; right: animals topically treated with NO-releasing PAAc/F127/GSNO hydrogel membranes.

\section{Conclusions}

PAAc/F127/GSNO are capable of prolonged spontaneous NO release during hydration. Topical application of the PAAc/F127/GSNO on wounds reduces inflammation and increases collagen deposition and orientation and has potential to accelerate wound healing.

\section{Acknowledgements}

Fundação de Amparo à Pesquisa do Estado de São Paulo,FAPESP (Project no 2015/17515-9)

\footnotetext{
${ }^{1}$ Witte MB, Barbul A. Role of nitric oxide in wound repair. Am J Surg. 2002 183:406-12.

${ }^{2}$ de Oliveira MG. S-nitrosothiols as platforms for topical nitric oxide delivery. Basic \& Clinical Pharmacology \& Toxicology, 2016, 118: 1-8.
} 